Disorders of the Nervous System

\title{
In Vivo Volume Dynamics of Dendritic Spines in the Neocortex of Wild-Type and Fmr1 KO Mice
}

(1) Kazuhiko Ishii, ${ }^{1,2}$ Akira Nagaoka, ${ }^{1}$ Yutaro Kishida, ${ }^{1,3}$ Hitoshi Okazaki, ${ }^{1,3}$ Sho Yagishita, ${ }^{1,3}$ Hasan Ucar, ${ }^{1,3}$ Noriko Takahashi, ${ }^{4}$ Nobuhito Saito, ${ }^{2}$ and ${ }^{1}$ Haruo Kasai ${ }^{1,3}$

https://doi.org/10.1523/ENEURO.0282-18.2018

${ }^{1}$ Laboratory of Structural Physiology, Center for Disease Biology and Integrative Medicine, Graduate School of Medicine, The University of Tokyo, Bunkyo-ku, Tokyo 113-0033, Japan , '2Department of Neurosurgery, Graduate School of Medicine, The University of Tokyo, Bunkyo-ku Tokyo 113-0033, Japan, ${ }^{3}$ International Research Center for Neurointelligence, The University of Tokyo Institutes for Advanced Study, The University of Tokyo, Bunkyo-ku, Tokyo 113-0033, Japan, and ${ }^{4}$ Department of Physiology, Kitasato University School of Medicine, Sagamihara 252-0374, Japan

\section{Visual Abstract}

Adult

Visual cortex

Wild type

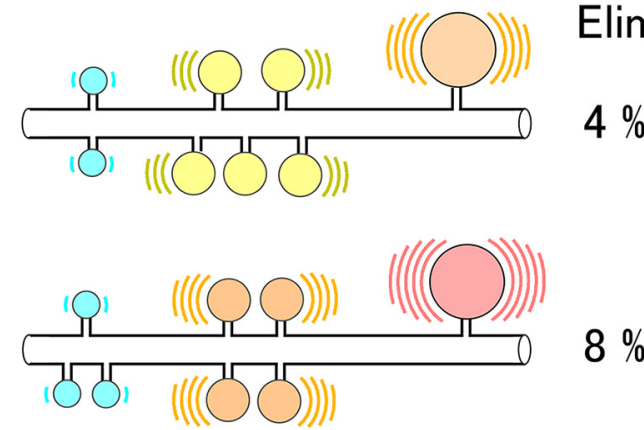

Elimination rate

Fmr1 KO

Dynamics

Young Hippocampus

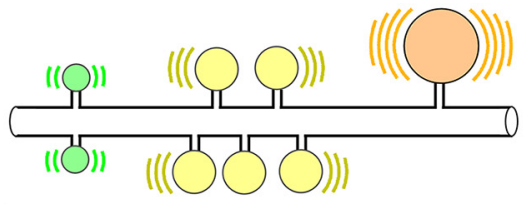

High

$12 \%$

Yasumatsu et al. (2008)

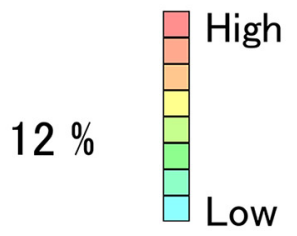

Slow dynamics (model)

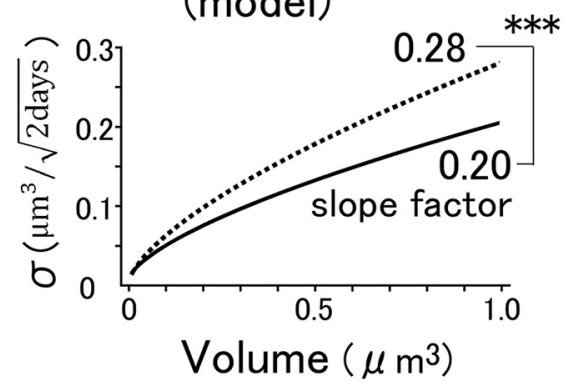

Spine volume distribution (model)

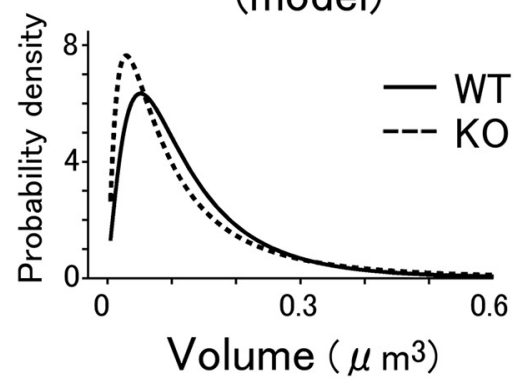




\section{Significance Statement}

Excitatory synapses are often formed at dendritic spines in projection neurons, and the spine volumes show strong correlations with synaptic connectivity. We studied the dynamics of spine volumes in vivo using a resonant scanner two-photon microscope and found that spine dynamics showed a distinctive dependence on spine volume in the adult neocortex, allowing for fewer fluctuations in these small spines than in the spines of young hippocampi. Moreover, the dynamics of spine volumes explained the greater turnover rate of spines and the smaller spine volume observed in a mouse model (Fmr1 knockout) of fragile X syndrome mental retardation than in wild-type mice. Our results indicate that the dynamics of spine volumes are abnormal in Fmr1 knock-out mice, even in adulthood.

Excitatory synapses are often formed at small protrusions of dendrite, called dendritic spines, in most projection neurons, and the spine-head volumes show strong correlations with synaptic connectivity. We examined the dynamics of spine volume in the adult mouse visual cortex using time-lapse in vivo two-photon imaging with a resonant Galvano scanner. Contrary to expectations, we found that the spines in the adult neocortex showed fluctuations to a similar degree as that observed in young hippocampal preparations, but there were systematic differences in how the dynamics were dependent on spine volumes, thus allowing for fewer fluctuations in small spines, which could account for the relatively low turnover rates of neocortical spines in vivo. We found that spine volumes fluctuated to a greater extent in a mouse model ( $F m r 1$ knockout) of fragile $\mathrm{X}$ mental retardation than in wild-type mice, and the spine turnover rates were also higher in Fmr1 knock-out mice. Such features of spine dynamics in Fmr1 knock-out mice could be represented by a single slope factor in our model. Our data and model indicate a small but significant change in the average spine volume and more eminent differences in the statistical distribution in Fmr1 knock-out mice even in adulthood, which reflects the abnormal in vivo dynamics of spine volumes.

Key words: ASD; autism; dendritic spine; fragile X mental retardation; synaptic plasticity; two-photon microscopy

\section{Introduction}

Excitatory glutamatergic synapses are formed at small protrusions of dendrites, called dendritic spines. As dendritic spines form synapses, their generation, enlargement, shrinkage, and elimination underlie the formation and maintenance of neuronal networks, and their head sizes strongly correlate with synaptic efficacy (Matsuzaki

Received July 12, 2018; accepted October 3, 2018; First published October 19, 2018.

The authors declare no competing financial interests.

Author contributions: K.I., A.N., and Y.K. performed research; K.I., H.O., S.Y., H.U., N.T., N.S., and H.K. analyzed data; K.I. and H.K. wrote the paper. This work was supported by Grants-in-Aid for Scientific Research (S; Grant 26221001 to H.K.), a Young Scientists (B; Grant 15K18333 to S.Y.), and Scientific Research on Innovative Areas (Grant $16 \mathrm{H} 06396$ to S.Y.) from The Japan Society for the Promotion of Science; Japan-EU Joint Workshop on Advanced Quantum Technology for Future Innovation (Grant JPMJCR1652 to H.K.) from the Japan Science and Technology Agency; Strategic Research Program for Brain Sciences Projects (17dm0107120h0002) from The Japan Agency for Medical Research and Development (to H.K.); and World Premier International Research Center Initiative from Ministry of Education, Culture, Sports, Science and Technology (to H.K.).

We thank H. Ohno, Y. Sakata, M. Asaumi, T. Nishikawa, and R. Yonemoto for technical assistance.

Correspondence should be addressed to Haruo Kasai, Laboratory of Structural Physiology, Center for Disease Biology and Integrative Medicine, Graduate School of Medicine, University of Tokyo, Bunkyo-ku, Tokyo, Japan. E-mail: hkasai@m.u-Tokyo.ac.jp.

https://doi.org/10.1523/ENEURO.0282-18.2018

Copyright (c) 2018 Ishii et al.

This is an open-access article distributed under the terms of the Creative Commons Attribution 4.0 International license, which permits unrestricted use, distribution and reproduction in any medium provided that the original work is properly attributed. et al., 2001, 2004; Noguchi et al., 2005; Béïque et al., 2006; Bhatt et al., 2009; Holtmaat and Svoboda, 2009; Kasai et al., 2010; Herring and Nicoll, 2016; Yasuda, 2017; Moyer and Zuo, 2018). The morphology and/or dynamics of dendritic spines have been found to be impaired in some psychiatric diseases (Fiala et al., 2002; Penzes et al., 2011), particularly autism spectrum disorder (ASD; Purpura, 1974). Fragile $X$ syndrome is one of the most prevalent monogenic forms of ASD and is caused by the expansion of CGG repeats in the Fmr1 gene, which encodes the Fragile $X$ Mental Retardation Protein (Consortium-D-BFX, 1994). In the fragile X mental retardation, dendritic spines are long, thin, and tortuous (Rudelli et al., 1985), suggesting that they are immature (He and Portera-Cailliau, 2013).

Fmr1 gene knock-out (KO) mice show abnormalities in learning and neuronal plasticity (Consortium-D-BFX, 1994; Comery et al., 1997; Cruz-Martín et al., 2010; He and Portera-Cailliau, 2013; Padmashri et al., 2013; Sidorov et al., 2013). These mice also show increases in the turnover rate of spine, which reflects the generation and elimination of spines (Pan et al., 2010; Padmashri et al., 2013; Nagaoka et al., 2016). Dendritic spines are found to be smaller in juvenile Fmr1 KO mice (Cruz-Martín et al., 2010; Pan et al., 2010; Swanger et al., 2011; He and Portera-Cailliau, 2013; Lauterborn et al., 2015), but the differences are not prominent in adult mice (Nimchinsky et al., 2001; Wijetunge et al., 2014). No previous study, however, has measured the changes in spine volumes and examined their correlations with the spine volume 
distributions in the mutant, because the quantification of volume dynamics was technically more demanding than that of spine turnover. Some groups have succeeded in the measurement of spine volume changes in wild-type (WT) mice (Yasumatsu et al., 2008; Loewenstein et al., 2011; Statman et al., 2014) and revealed that such dynamics provide a good basis for the volume distribution and turnover of spines using mathematical models with stochastic processes (random walks).

Here, we investigated the differences in the dynamics of dendritic spines in WT and Fmr1 $\mathrm{KO}$ adult mice in vivo. We used a two-photon microscope equipped with a resonant scanner to overcome the limitation of the inherent pulsations in brains in vivo. We applied the same model that was developed for the rat hippocampal slice preparations in vitro (Yasumatsu et al., 2008). We found that the spine fluctuations depended on the volume of spines in the adult neocortex, which differs from what is observed in the young hippocampus, which could account for the relative stability of the spines in the neocortex. It could also explain the difficulty in detecting the difference in the average spine volumes between adult WT and Fmr1 KO mice in previous reports (Nimchinsky et al., 2001; Wijetunge et al., 2014). Our study clarified the critical impairments in spine dynamics and other spine parameters in Fmr1 KO mice even in adulthood.

\section{Materials and Methods}

\section{Animals}

A colony of homozygous transgenic mice expressing a green fluorescent protein (GFP) under the control of the Thy1 promoter (Thy1-GFP M-line mice) was generated for the present study. B6.129P2-Fmr1 ${ }^{\mathrm{tm} 1 \mathrm{Cg}} / \mathrm{J}$ (The Jackson Laboratory) KO female mice $\left(F m r 1^{+/-}\right.$) were crossed with males homozygous for Thy1-GFP to generate GFPFmr1-/y mice. Adult male mice $>2$ months of age were anesthetized with isoflurane $(4.5 \%$ for induction; $0.8-$ $1.5 \%$ for maintenance) during the operation. The level of anesthesia was assessed by monitoring the tail-pinch reflex. The administration of $20 \%$ mannitol $(20 \mu \mathrm{l} / \mathrm{g}$ body weight) was performed intraperitoneally, while ketoprofen ( $2 \mu \mathrm{l} / \mathrm{g}$ body weight) was administered subcutaneously. A head plate with a 5-mm-diameter hole was fixed to the skull using dental cement (Fuji Lute BC, GC). A small craniotomy (diameter, $2.7 \mathrm{~mm}$ ) was performed over the left visual cortex based on stereotaxic coordinates (posterior, $3.0 \mathrm{~mm}$ from the bregma; lateral, $2.5 \mathrm{~mm}$ from the bregma; Paxinos, 2001), which were confirmed by intrinsic signal imaging. The craniotomy was performed using a trephine drill (catalog \#224RF-027, Meisinger) fixed to a stereotaxic instrument. A circular glass (diameter, $2.7 \mathrm{~mm}$; Matsunami) was fixed to the cranial window using the dental cement (Fuji Lute BC, GC) and a dental acrylic device (ADFA, Shofu) to the cranial window.

Imaging experiments were performed at least $1 \mathrm{~d}$ after the surgery and repeated every other day for 3-23 d (2-12 imaging sessions) as long as the conditions of the animal, cranial window, brain, and dendrites remained stable. Data were pooled and analyzed together. The most frequent contiguous imaging duration was $5 \mathrm{~d}$. Examples of images obtained in this duration are shown in Figure 1, $B$ and $C$, with the corresponding spine volume time course shown in Figure 2, $A$ and $B$. After imaging, mice were returned to their respective cages. Five $\mathrm{KO}$ mice and five WT (littermates) mice were examined, and one to five dendrites were imaged for each mouse. Images were obtained from five other mice in a similar manner at intervals of $10 \mathrm{~min}$. All animal procedures were performed in accordance with the regulations of The University of Tokyo Animal Care Committee.

\section{Two-photon imaging}

Two-photon imaging was performed using an upright microscope (models BX61WI and FV1000-MP, Olympus) equipped with a resonant scanner and a GaAsP detector with a $25 \times(1.05$ numerical aperture; catalog \#XLPLN25XWMP2, Olympus) water-immersion lens. A Tisapphire laser (Mai-Tai-DS-HP, SpectraPhysics) was tuned to $950 \mathrm{~nm}$ wavelength for the excitation of the GFP. The average excitation power was maintained at $<40 \mathrm{~mW}$ under the objective. The power was selected to avoid saturating the fluorescence of dendrites. Mice were anesthetized with a $0.8-1.5 \%$ isoflurane-oxygen mixture (Univentor 400 anesthesia unit, Univentor), and the body temperature was maintained at $37^{\circ} \mathrm{C}$ using a heating pad. During the image acquisition, mice were restrained using a head plate. Image stacks were obtained from the apical dendritic tufts of layer $V$ pyramidal in the layer I $(20-70 \mu \mathrm{m}$ from the pial surface), and, typically, 20-40 frames (512 $\times$ 512 pixels; $0.124 \mu \mathrm{m} /$ pixel; $17 \mathrm{~ms} /$ frame) per stack were obtained at an interval of $0.4 \mu \mathrm{m}$ along the $z$-axis. Each dendrite was traced to the soma to confirm its layer of origin.

\section{Measurements of spine dynamics}

Z-stack images were aligned using StackReg in ImageJ (RRID:SCR_003070), and summed over the $z$-direction. Only spines that were well separated from the dendrite were included in the analysis. A region of interest (ROI) surrounding the spine head was chosen manually, and another ROI was placed at the same distance from the dendrite where there were no spines, to obtain the background fluorescence level, which was then subtracted from the fluorescence of the ROI over the spine. The total fluorescence $(F)$ of a spine head was considered to be related to spine-head volume $(V)$, assuming that GFP homogeneously filled the volume of the spine, as previously described (Matsuzaki et al., 2004, Supplementary Methods). To this end, the largest and most spherical spine on the dendrite was selected from the data obtained in an early imaging session of each dendrite, and the one-dimensional fluorescence profile across the center of the spine head was measured. This was fitted by the following equation for the fluorescence intensity profile of spherical objects of radius $R$ (Eqn. 1):

$$
\frac{A}{(2 \pi)^{3 / 2} \sigma_{x}^{2} \sigma_{z}} \iiint_{x^{2}+y^{2}+z^{2}<R^{2}} d x d y d z \int d z^{\prime} e^{-\left[\frac{(x-r)^{2}}{2 \sigma_{x}^{2}}+\frac{y^{2}}{2 \sigma_{x}^{2}}+\frac{\left(z-z^{\prime}\right)^{2}}{2 \sigma_{z}^{2}}\right],}
$$


A
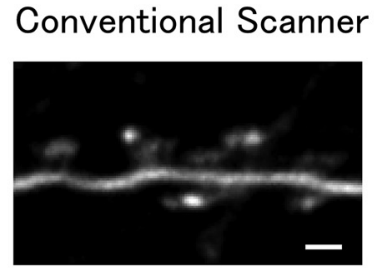

B

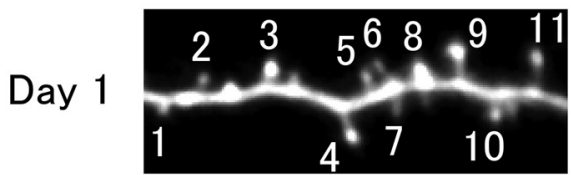

WT

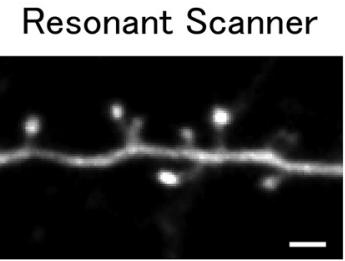

C $\quad F m r 1 \mathrm{KO}$
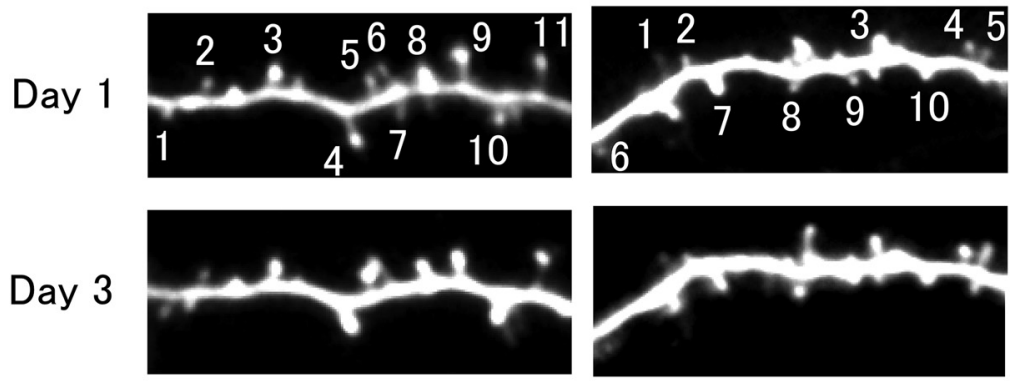

Day 5
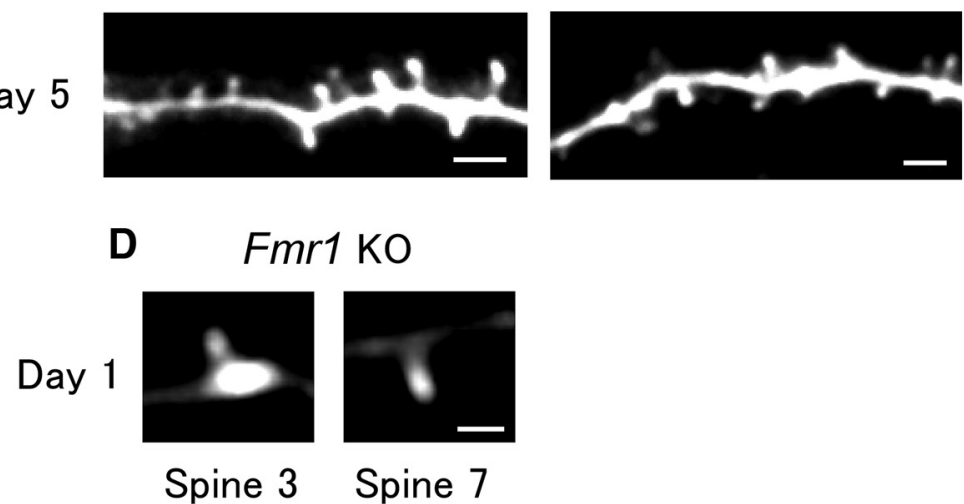

Figure 1. Apical dendritic spines of M-line mice in the mouse visual cortex imaged at an interval of $2 \mathrm{~d}$. $\boldsymbol{A}$, Sample images of a mouse with considerable pulsation artifacts obtained with conventional and resonant Galvano scanners. The resonant scanner was relatively robust in response to motion artifacts. Scale bar, $2 \mu \mathrm{m}$. $\boldsymbol{B}, \boldsymbol{C}$, Examples of a time-lapse series of dendrite images from WT $(\boldsymbol{B})$ and $\mathrm{KO}(\boldsymbol{C})$ mice. Spine numbers correspond to the respective traces in the volume-time courses in Figure 2, $A$ and $B$. Scale bar, $3 \mu \mathrm{m}$. $\boldsymbol{D}$, Spines 3 and 7 in $\boldsymbol{C}$ are displayed at a different brightness level from that used in $\boldsymbol{C}$. Scale bar, $1 \mu \mathrm{m}$.

where $A$ represents the intensity at the center $(r=0)$ of the sphere. Point spread functions were estimated to be 0.56 $\mu \mathrm{m}$ full-width at half-maximum $\left(\sigma_{x}=0.24 \mu \mathrm{m}\right)$ laterally and $2.1 \mu \mathrm{m}\left(\sigma_{z}=0.91 \mu \mathrm{m}\right)$ axially based on the images of the smallest structures such as filopodia in our own experiments in vivo. The volume of the spine was thus calculated as $V=4 / 3 \pi R^{3}$, and its ratio (V/F) to the total fluorescence was used for calculating spine volume from its total fluorescence in the same field for all subsequent imaging sessions. This conversion coefficient could be used to estimate spine volumes irrespective of their fine structures. To correct for day-to-day variation in the expression of GFP, total fluorescence of the dendritic region was recorded for each sample on each day. Estimates of spine-head volume were corrected for this variation, which was usually $<10 \%$ per day (range, $0.3-25 \%$; Yasumatsu et al., 2008).

Filopodia (protrusions with head diameter/neck diameter ratio, <1.2; length/neck diameter ratio, >3) were excluded from the analysis. For the measurement of elimination rates, original images were carefully examined to confirm the elimination to avoid misleading interpretations of spine elimination for spines moving behind dendrites or overlapping with a neighbor. Images not bright and clear enough to enable unambiguous distinction were excluded from the measurement of elimination rates.

\section{Mean and SD of changes in spine-head volume}

The mean change in spine-head volume $[\mu(V)]$ and the SD of this change $[\sigma(V)]$ were calculated per $2 \mathrm{~d}$ using the following equations (Eqn. 2, 3):

$$
\begin{gathered}
\sigma^{2}\left(R_{j}\right)=\sum_{i, k \mid V_{k}(i) \in R_{j}}\left(V_{k}(i+1)-V_{k}(i)-\mu\left(R_{j}\right)\right)^{2} / N_{j} \\
\mu\left(R_{j}\right)=\sum_{i, k \mid V_{k}(i) \in R_{j}}\left(V_{k}(i+1)-V_{k}(i)\right) / N_{j},
\end{gathered}
$$

where each value of $\mu(V)$ and $\sigma(V)$ was obtained for individual imaging sessions $(i=1,2, \ldots)$ and where spines $(k=1,2, \ldots)$ had volumes of $V_{k}(i)$ falling within each of the 
A

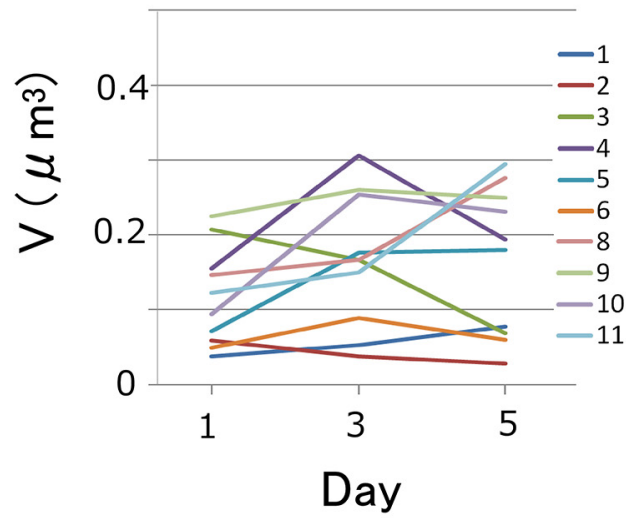

B $\quad F m r 1 \mathrm{KO}$

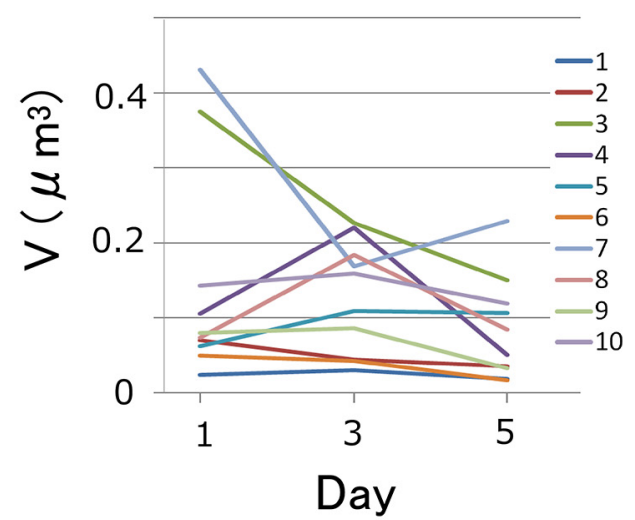

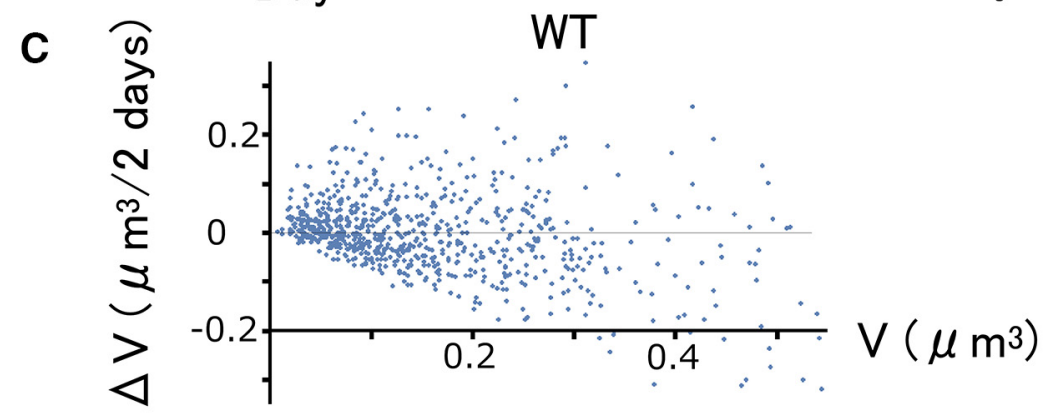

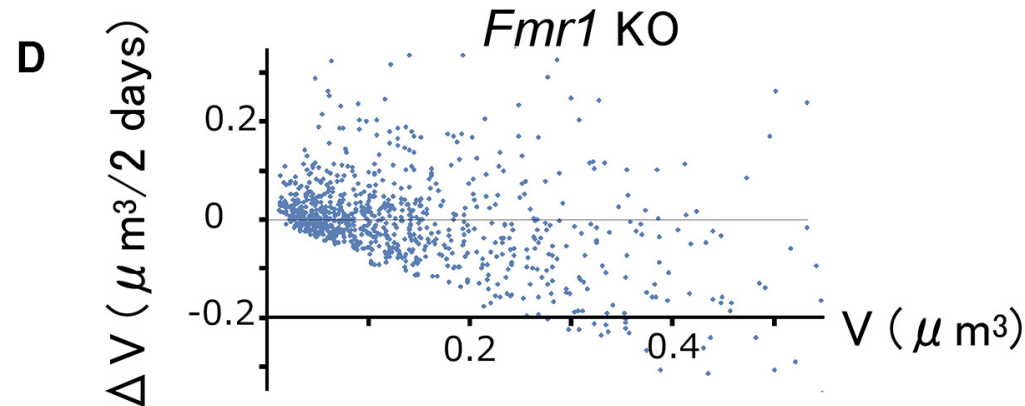

Figure 2. Changes in spine volumes in the mouse visual cortex per $2 \mathrm{~d}$. $\boldsymbol{A}, \boldsymbol{B}$, Time course of spine volume changes for the spines shown in Figure 1, $B$ and $C$. Trace numbers correspond to the spines numbered in Figure $1, B$ and $C$. $\boldsymbol{C}, \boldsymbol{D}$, Spine-head volume changes $(\Delta V)$ per $2 \mathrm{~d}$ in WT $(\boldsymbol{C})$ and $F m r 1 \mathrm{KO}(\boldsymbol{D})$ mice. Data were obtained from 754 spines from 15 dendrites in five WT mice and from 878 spines from 20 dendrites in five $\mathrm{KO}$ mice.

volume bins $R_{j}(j=1,2, \ldots, 24) . N_{j}$ is the total number of spines satisfying $V_{k}(i) \in R_{j}$.

The values of $\sigma$ defined by Equation 2 included the fast fluctuations by the rapid actin dynamics and measurement errors, which should be treated differently from the long-term behaviors of spines. We thus obtained such rapid fluctuations, $\sigma_{\text {fast }}$, by imaging spine volumes every $10 \mathrm{~min}$ for $1 \mathrm{~h}$ (see Fig. 4). The mean values $(\mu)$ were zero, consistent with the nature of the fast fluctuations, and $\sigma_{\text {fast }}$ could be fitted by the following equation (Fig. $3 E, F$, dotted lines; and see Fig. 5, dotted lines) (Eqn. 4):

$$
\sigma_{\text {fast }}=0.115 \mathrm{~V}^{2 / 3}+0.0051 \text {. }
$$

For simplicity, we expressed the values as the SD of the sample in Figure 5, as the means were zero, and the variation did not depend on the sampling interval by definition (Matsuzaki et al., 2004; Yasumatsu et al., 2008).
The total dynamics were the sum of these fast dynamics and the slow dynamics $\left(\sigma_{\text {slow }}\right)$ that determined the longterm behaviors of the spine, as follows (Eqn. 5):

$$
\sigma^{2}=\sigma_{\text {fast }}^{2}+\sigma_{\text {slow. }}^{2}
$$

The dynamics of $\sigma$ were plotted against $V$ in a double logarithmic plot, as seen in Figure $3, C$ and $D$. We noted that the slope was less than one (red line), and nearly two-thirds (black line). Therefore, in the following graphs, $\mu$ and $\sigma$ were plotted against $V^{2 / 3}$. A regression line for the slow dynamics $\left(\sigma_{\text {slow }}\right)$ calculated using Equation 5 , and the experimentally obtained $\sigma$ and $\sigma_{\text {fast }}$ was plotted against $V^{2 / 3}$, as seen in Figure $3 E$ (dashed line), and was expressed as follows (Eqn. 6):

$$
\begin{aligned}
\sigma_{\text {slow }}(V) & =0.198\left(V^{2 / 3}-0.06\right)+0.020 \\
& =0.198 V^{2 / 3}+0.0081 .
\end{aligned}
$$


A

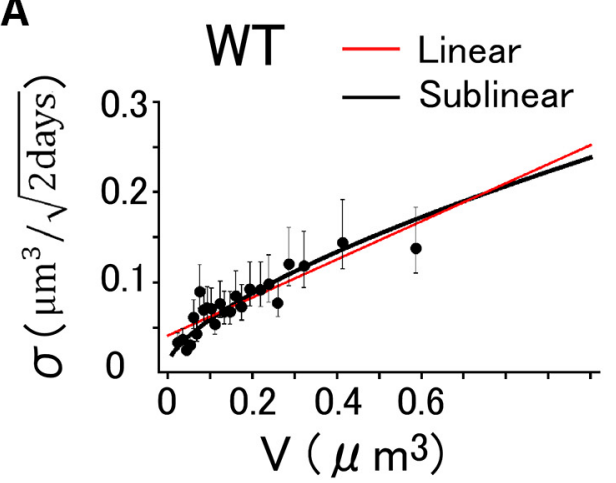

C

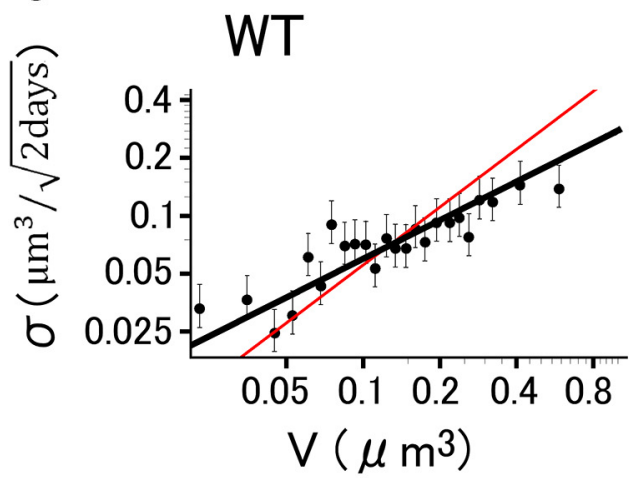

E

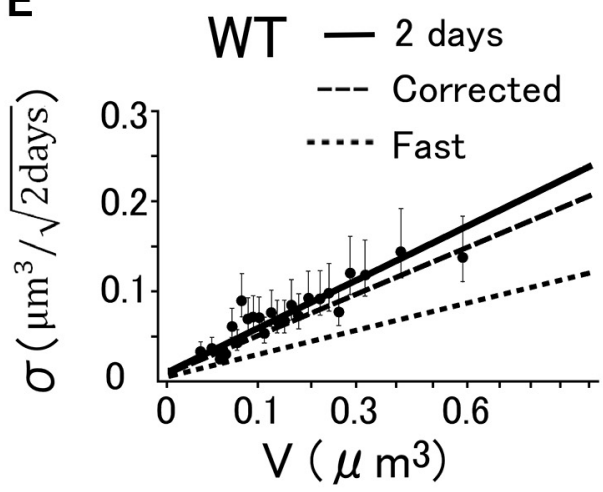

G

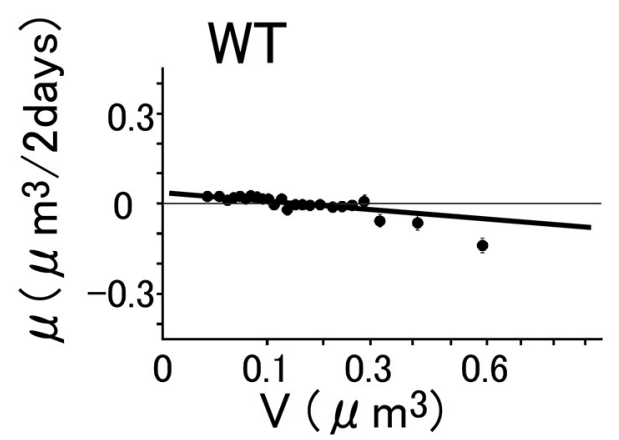

B

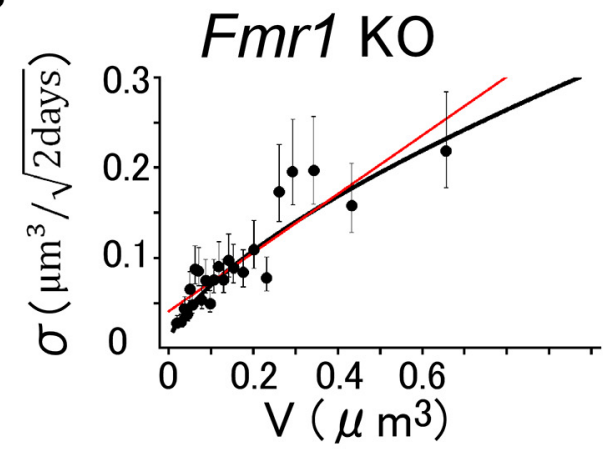

D

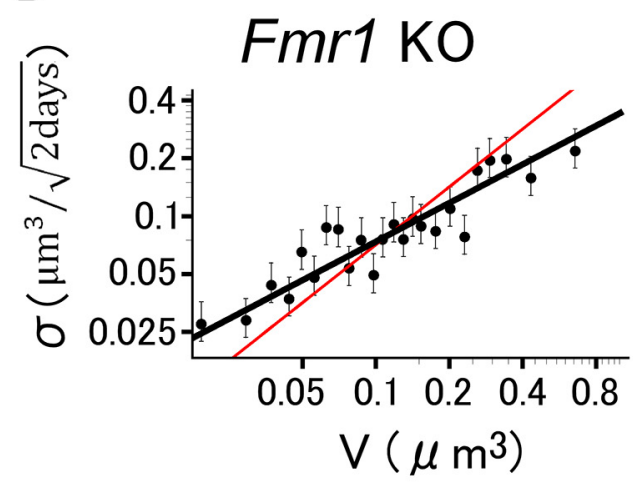

F

Fmr1 KO

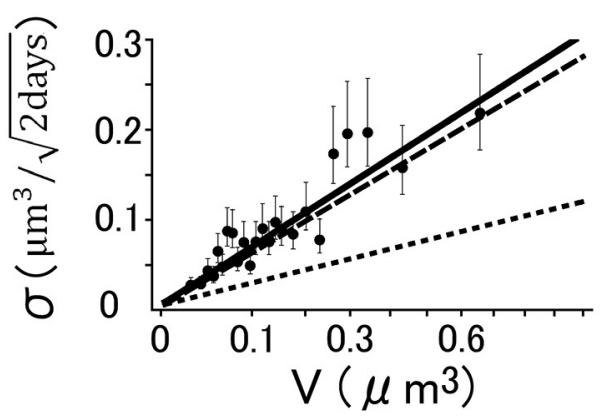

H

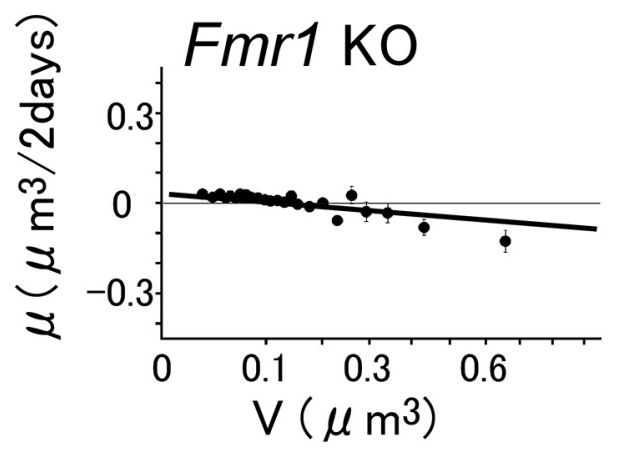

Figure 3. Slow dynamics of spine-head volume measured per $2 \mathrm{~d}$. $\boldsymbol{A}, \boldsymbol{B}$, The dynamics of spine-head volumes in WT (A) and KO (B) mice plotted against $V$. Each plotted point represents the SD of spine-head volume changes in 32 pooled spines of similar volume. Error bars represent $95 \%$ confidence intervals of the estimated SD. The red lines represent the least squares fits. The red line for WT mice $(\boldsymbol{A})$ is represented by $0.21 \mathrm{~V}^{2 / 3}+0.041$, and for KO mice $(\boldsymbol{B})$ it is represented by $0.32 \mathrm{~V}^{/ 3}+0.041$. Note that the values of $\sigma$ were below the red lines for small spines. $\boldsymbol{C}, \boldsymbol{D}$, Double logarithmic plots of the dynamics of spine-head volumes shown in $\boldsymbol{A}$ and $\boldsymbol{B}$. Each plotted point represents the SD of spine-head volume changes in 32 pooled spines of similar volume. Error bars represent $95 \%$ 


\section{continued}

confidence intervals of the estimated SD. The black lines with a slope of two-thirds fit well with the data. The red lines are reference lines with a slope of $1 . \boldsymbol{E}, \boldsymbol{F}$, Slow spine-head volume changes in WT $(\boldsymbol{E})$ and $F m r 1$ KO $(\boldsymbol{F})$ mice plotted against $V^{2 / 3}$ (note that the numbers and scales on the $x$-axis represent $V$ ). Each plotted point represents the SD of spine-head volume changes in 32 pooled spines of similar volume. The solid line is a fit to the data, and can be decomposed into a slow component (dashed lines; Eq. 6 , confidence interval of the slope 0.18-0.22) and a fast component, obtained using measurements at 10 min intervals (dotted lines; Eq. 4) for $\boldsymbol{E}$, and Equation 7 (95\% confidence interval of the slope $0.24-0.32)$ for $\boldsymbol{F}$. The slope of the fitted line for KO mice is significantly larger than that for WT mice (generalized linear model, $t$ statistic $=-3.67, p=0.0006^{\mathrm{a}}$ ). $\boldsymbol{G}, \boldsymbol{H}$, The drift of the spine-head volume changes in WT $(\boldsymbol{G})$ and $F m r 1 \mathrm{KO}(\boldsymbol{H})$ mice plotted against the initial $V$ value. The abscissa is linear in $V^{2 / 3}$, while the numbers and scales represent $V$ as in $\boldsymbol{E}$ and $\boldsymbol{F}$. Each plotted point represents the mean of spine-head volume changes in 32 pooled spines of similar volume. Error bars represent the SEM. The lines are the same for the pooled data in WT and KO mice (Eq. 8).

For Fmr1 KO mice, it was as follows (Eqn. 7):

$$
\begin{aligned}
\sigma_{\text {slow }}(V) & =0.278\left(V^{2 / 3}-0.06\right)+0.020 \\
& =0.278 V^{2 / 3}+0.0033 .
\end{aligned}
$$

The slow drift $\mu$ (per day) was obtained in a similar manner in both WT and Fmr1 KO mice, as shown below (Eqn. 8):

$$
\mu(V)=-0.12 V^{2 / 3}+0.029 .
$$

To reassure that the operation itself did not affect the results, the analysis was performed excluding imaging data from day 1 postsurgery. The slope factors were 0.192 (generalized linear model, $p=0.95$, compared with 0.198 in Eq. 6) and $0.279(p=0.95$, compared with 0.278 in Eq. 7). They are almost the same as the original analysis results, and the slope factor for $\mathrm{KO}$ is significantly larger than for WT mice as before (generalized linear model, $p=$ $0.0003^{a}$ ). Equation 8 remained the same.

\section{Model of spine dynamics and the solution of the Langevin equation}

The slow dynamics of $V$ were modeled as the random variation defined using a Brownian motion (Eq. 9) within the range between the minimal $\left(V_{\min }\right)$ and maximal $\left(V_{\max }\right)$ values of 0.01 and $1.0 \mu \mathrm{m}^{3}$, respectively, because spines with volume $<0.01$ or $>1.0 \mu \mathrm{m}^{3}$ were rare. Brownian motion $(M)$ is the simplest random process, in which the mean is zero, but the variance is proportional to time. Using Brownian motion, a more general form of random processes with a mean $\mu(V)$ and variance $\sigma(V)$, which depend on $V$, can be described by the following Langevin equation (Eqn. 9):

$$
\frac{d V(t)}{d t}=\sigma(V(t)) \frac{d W(t)}{d t}+\mu(V(t))
$$

where $W(t)$ is the standard Brownian motion with a variance of 1 per $2 \mathrm{~d}$. Following this, the transition probability density $p(V, t \mid y)$ of the spine volume, which was $V$ and $y$ at time $t$ and 0 , respectively, satisfied the Fokker-Planck equation, if we performed stochastic integration according to Ito's definition, as follows (Eqn. 10):

$$
\begin{gathered}
\frac{\partial p(V, t \mid y)}{\partial t}=\frac{1}{2} \frac{\partial^{2}}{\partial V^{2}}\left[\sigma^{2}(V) p(V, t \mid y)\right] \\
-\frac{\partial}{\partial V}[\mu(V) p(V, t \mid y)] .
\end{gathered}
$$

If a spine with the value of $V$ bounces or disappears at the boundary, the boundary would be termed as reflecting or absorbing, respectively. How these boundaries are mathematically realized can be seen in the Mathematica (RRID:SCR_014448) notebook (Yasumatsu et al., 2008). The stationary solution of the Fokker-Planck equation can be derived directly, as follows, when a reflecting boundary condition is assumed at both $V_{\min }$ and $V_{\max }$ (Eqn. 11):

$$
f(V)=\frac{C}{\sigma^{2}(V)} \operatorname{Exp}\left[\int \frac{2 \mu(V)}{\sigma^{2}(V)} d V\right]
$$

where $C$ is a constant for normalization (Risken, 1984). The values of $\sigma(V)$ and $\mu(V)$ were obtained from the experiments (Eqs. 6-8). Calculated $f(V)$ was plotted to obtain the stationary distribution function of the spine volume, which is shown in Figure 7A.

The elimination rate of spines in $T$ days was calculated as follows (Eqn. 12):

$$
E(T, f)=1-\int_{V_{\min }}^{V_{\max }} \int_{V_{\min }}^{V_{\max }} p(V, T \mid y) f(y) d y d V,
$$

where the stationary distribution $f(y)$ as given above is assumed as the initial distribution and the absorbing boundary is set at $V=V_{\min }$.

\section{Statistical analyses}

A generalized linear model was used to compare the slopes of the fitted line in the plots of $\sigma$ against $V^{/ 3}$ (Fig. $3 E, F)$. Spine elimination rates were compared using a Mann-Whitney $U$ test (see Fig. $6 C$ ). The comparison of spine volume distribution was performed using a Kolmogorov-Smirnov test (see Fig. 7C; Table 1).

\section{Code accessibility}

The Mathematica notebook file of this study is available at https://doi.org/10.1523/ENEURO.0282-18.2018.f1-1. All code is also available as Extended Data 1.

\section{Table 1: Statistics}

\begin{tabular}{lllc}
\hline & Data structure & \multicolumn{1}{c}{ Type of test } & Power \\
a & Not normal & Generalized linear model & Not applicable \\
b & Not normal & Mann-Whitney $U$ test & Not applicable \\
c & Not normal & Kolmogorov-Smirnov test & Not applicable
\end{tabular}




\section{Results}

\section{Dynamics of spine-head volumes}

In vivo time-lapse imaging of the dendritic spines of the adult mouse visual cortex using two-photon microscopy was performed over 1 week to observe the longitudinal behavior of dendritic spines. A small glass window was made in the skull above the visual cortex of adult Thy1GFP M mice, in which GFP was sparsely expressed, but was mainly found in layer $\mathrm{V}$ pyramidal neurons. A resonant scanner made it possible to obtain clearer images than those obtained with a conventional Galvano scanner in two-photon imaging (Fig. 1A). In fact, even in samples with considerable motion artifacts owing to heartbeats and respiration, the images were clear enough for spine volume measurements (Fig. 1A).

The analysis was restricted to layer I apical dendrites of layer $\mathrm{V}$ pyramidal neurons. Filopodia were excluded from the analysis (Materials and Methods), because they are thought to lack synaptic contacts and are expected to behave differently. Dendrites were imaged repeatedly at an interval of $2 \mathrm{~d}$ in WT and KO mice (Fig. 1B,C). Magnified images of two spines in Figure $1 C$ are displayed in Figure $1 D$, showing that their heads are well defined. Spine volume was calculated from the images as explained in the Materials and Methods (Fig. 2A,B). Small spines were more likely to become larger (Fig. 2C, $D$, representing 754 spines from 15 dendrites in five WT mice and 878 spines from 20 dendrites in five $\mathrm{KO}$ mice), and large spines showed larger fluctuations in size, as observed in previous studies (Yasumatsu et al., 2008; Minerbi et al., 2009).

\section{Quantitative analysis of spine-head volume dynamics}

Data from spines with similar volumes were pooled as explained in the Materials and Methods, and then the SD (Eq. 2) and the mean (Eq. 3) of the absolute volume changes per $2 \mathrm{~d}$ were calculated for quantitative evaluations. The dynamics expressed as the SD $(\sigma)$ increased monotonically with increasing values of $V$, but the increase was sublinearly dependent on $V$ (Fig. $3 A$ ), unlike what is observed in the young hippocampus (Yasumatsu et al., 2008). If we simply fit a line to $\sigma$ (Fig. $3 A, B$, red lines), the linear slope factors were 0.21 and 0.32 for WT and Fmr1 KO mice, respectively. The linear fits, however, showed relatively large errors for small spines $(<0.06$ $\mu \mathrm{m}^{3}$ ), indicating that the small spines fluctuated less than was linearly predicted using $V$ for all spines.

A double logarithmic plot showed that the sublinearity was $V^{0.6}$ (Fig. $3 C, D$ ). Therefore, a value of two-thirds was chosen to represent this sublinearity, to allow an alternative interpretation of $V^{2 / 3}$, instead of the volume $V$, as the surface area of the spine. Subsequently, $\sigma$ was plotted against $V^{2 / 3}$ (Fig. $3 E, F$, filled circles). Since the behavior of very small spines has an important influence on the spine turnover, we anchored the line to a point at $V=0.015$ (the median value of the smallest bin) and $\sigma=0.02$ (average value of $\sigma$ for the smallest bin), and obtained a suitable slope for the straight line ( $\sigma$ vs $\left.V^{2 / 3}\right)$ from the observed data, as shown with the solid lines. The values of $\sigma$ remained positive near $V=0$, corresponding to the observations that even very small spines showed a certain amount of dynamics, as seen in the scatter chart (Fig. $2 C, D$ ). The difference in the slope between WT and KO mice is statistically significant (generalized linear model, $p$ $=0.0006^{\mathrm{a}}$ ), indicating that the volume dynamics of spines are significantly greater in KO than in WT mice.

Similarly, the mean of the spine volume that changed per $2 \mathrm{~d}$ ( $\mu$, drift) was plotted against $V^{2 / 3}$ (Fig. 3G,H). The values of $\mu$ decreased monotonously with an increase in the spine volume and showed a value of zero at $V=0.12$. Spines smaller than this volume tended to increase in size, as per the scatter chart. Since the means of spine volume changes were similar between the WT and KO mice (Fig. 3, compare $G, H$ ), they were fitted with the same equation (Equation 8).

\section{Fast and slow dynamics}

The dendritic spines displayed fast fluctuations, which occurred independently of the slow dynamics that determined spine elimination and volume distributions. To estimate the fast dynamics in the adult mice, we performed time-lapse imaging with an interval of $10 \mathrm{~min}$ for $1 \mathrm{~h}$ (Fig. $4 A, B)$. These observations cannot simply reflect measurement errors (Mysore et al., 2007; Yasumatsu et al., 2008), because such fluctuations were not observed in the fixed brain preparations even after a 2 d interval (Fig. 4C; generalized linear model, $p=0.00002^{\mathrm{a}}$ ). Since the fast dynamics (Fig. 4D,E) were similar between the WT and KO mice (Fig. 5, compare $A, B$ ), they were fitted with the same equation (Equation 4). The means of the spine volume changes were negligible for both the WT and KO mice (Fig. $5 C, D$ ), and were represented by zero. Since these fluctuations mainly reflect actin-dependent rapid fluctuations (Honkura et al., 2008), we simply expressed these using variances and means (Fig. $5 A-D$ ). Using the fast dynamics and Equation 5, the slow dynamics were obtained with Equations 6 and 7, as shown in the dashed lines in Figure 3, $E$ and $F$.

\section{Spine turnovers}

Spine elimination was measured using time-lapse imaging with an interval of $2 \mathrm{~d}$, and was expressed as the percentage of the spines that disappeared in the observation period. Figure $6, A(\mathrm{WT})$ and $B(\mathrm{KO})$, shows examples of the spine eliminations occurring in the $2 \mathrm{~d}$ interval. The elimination rates observed were 4.1\% (WT) and $8.5 \%$ (KO) per $2 \mathrm{~d}$ (Fig. 6C; Mann-Whitney $U$ test, $p=0.08^{\mathrm{b}}$ ), which is consistent with a previous observation (Nagaoka et al., 2016) that the elimination rate was significantly higher in KO mice (Steel's test, $p<0.01$ ).

Predictions for spine elimination rates using the model were performed by replacing the boundary condition at the minimum spine volume from a reflecting boundary to an absorbing boundary, as explained in the Materials and Methods. The predicted rates could then be compared with the measured elimination rates. Brownian motion describes the behavior of a timedependent random variable, $V(t)$, with an SD, $\sigma(V)$, and a drift, $\mu(V)$, as the stochastic process, as per Equations 9 and 10. Using Equation 12, the elimination rate for WT 
A

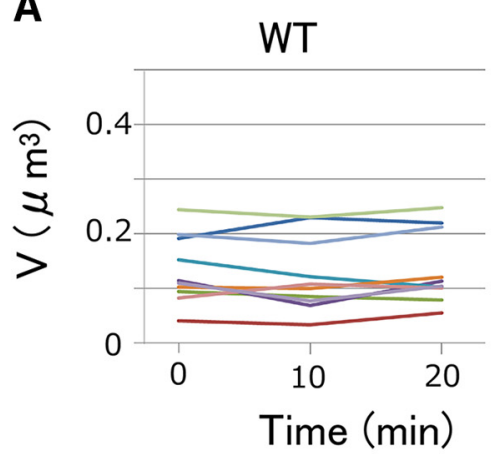

D

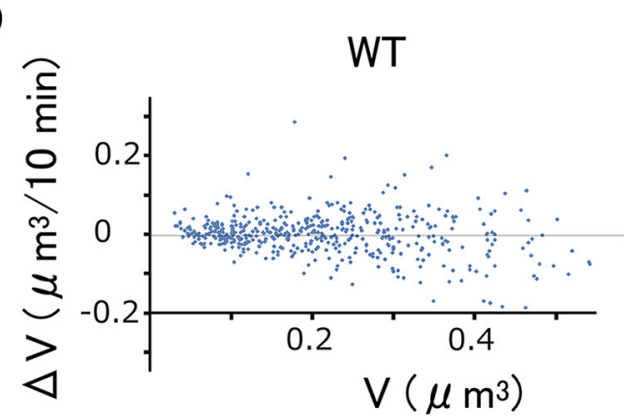

B

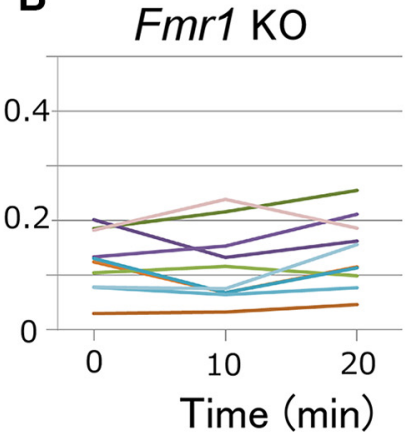

E

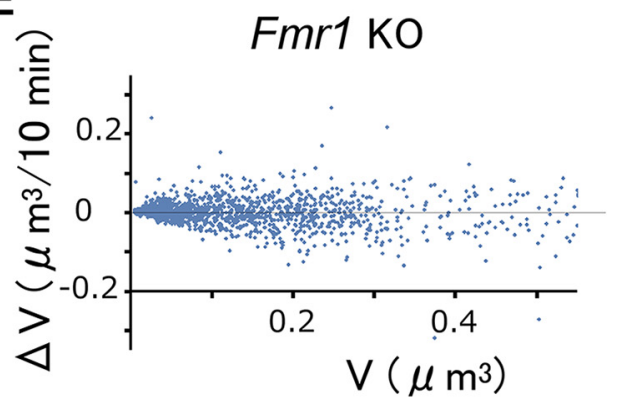

C

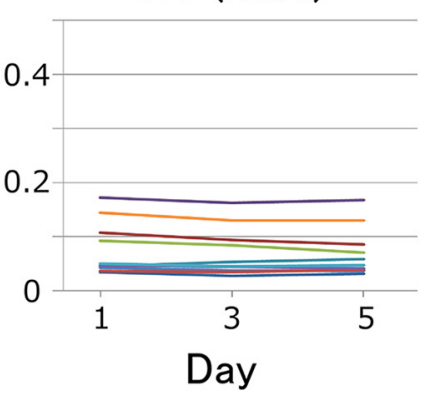

Day

Figure 4. Fast volume changes in apical dendritic spines in the mouse visual cortex. $\boldsymbol{A}, \boldsymbol{B}$, Spine-head volume changes in WT (A) and KO (B) mice measured at an interval of $10 \mathrm{~min}$. C, Spine-head volume changes in a fixed sample imaged at an interval of $2 \mathrm{~d}$. $\boldsymbol{D}, \boldsymbol{E}$, Head volume changes $(\Delta V$ per 10 min in WT $(\boldsymbol{D})$ and $F m r 1 \mathrm{KO}(\boldsymbol{E})$ mice. Data were obtained from 475 spines from two dendrites in two WT mice, and from 1433 spines from four dendrites in three KO mice.

A

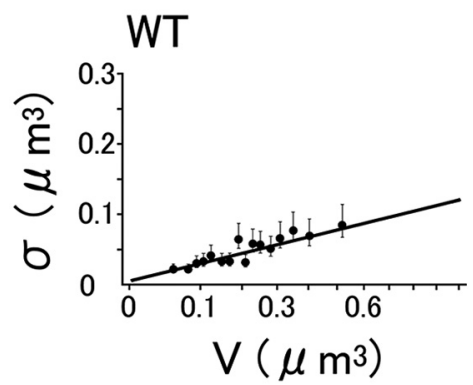

C

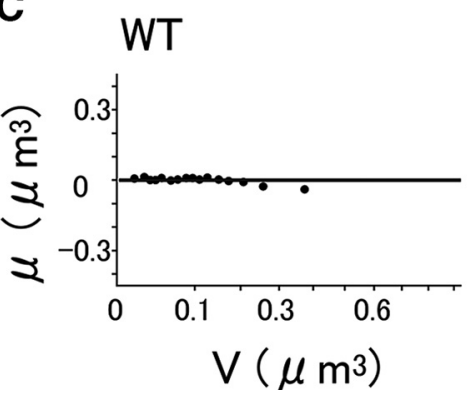

B
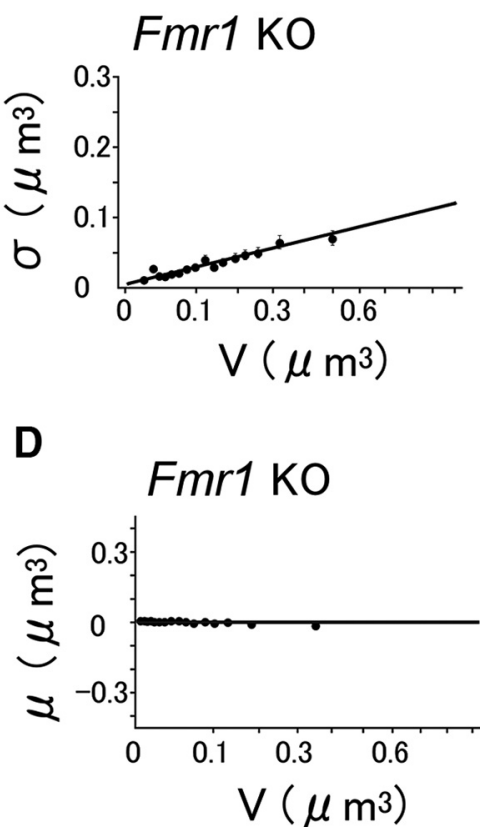

Figure 5. Fast dynamics of spine-head volume. $\boldsymbol{A}, \boldsymbol{B}$, The fast component of the dynamics of spine-head volume in WT (A) and KO $(B)$ mice plotted against $V^{2 / 3}$. Each plotted point represents the SD of spine-head volume changes in 30 pooled spines of similar volume. Error bars represent $95 \%$ confidence intervals of the estimated SD. The line represents the least squares fit of the pooled data for WT and KO (Eq. 4). C, D, Fast component of the drift of spine-head volume in WT (C) and KO (D) mice plotted against $V^{2 / 3}$. Each point represents the mean of spine volume changes in 30 spines of similar volume. Error bars represent SEM values. Because the drifts were very small, they were fitted by zero (line). 


\section{A}

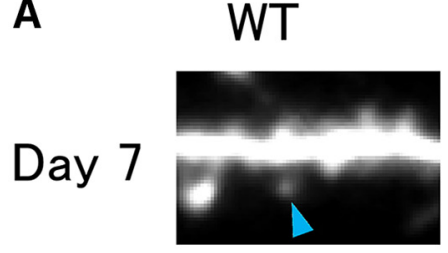

Day 9

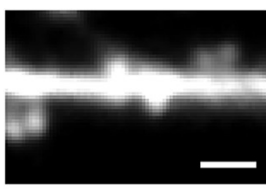

B
Day 3

Fmr1 KO

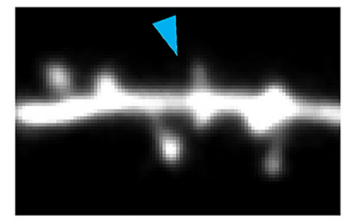

Day 5

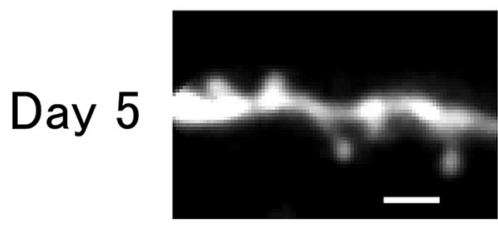

C

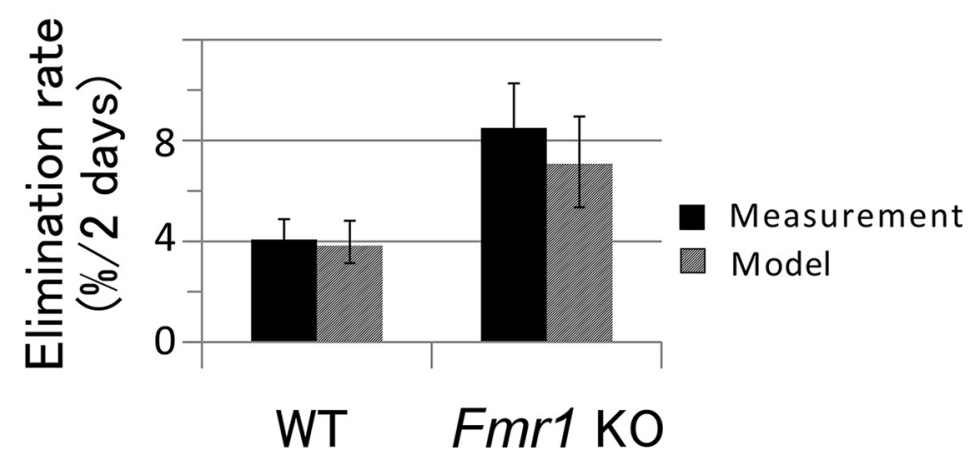

Figure 6. Elimination of dendritic spines per 2 d. $\boldsymbol{A}, \boldsymbol{B}$, Example of a time-lapse series of images of a dendrite in WT $(\boldsymbol{A})$ and KO $(\boldsymbol{B})$ mice at an interval of $2 \mathrm{~d}$. Arrowheads indicate eliminated spines. Scale bar, $2 \mu \mathrm{m}$. $\boldsymbol{C}$, The elimination rates of dendritic spines per $2 \mathrm{~d}$. Solid bars represent measurement data. Error bars represent SEM values from 6 and 12 dendrites for WT and Fmr1 KO mice, respectively. KO mice seemed to have a higher elimination rate, although the difference was not significant (Mann-Whitney $U$ test, $U=17, p=0.08^{\mathrm{b}}$ ). Hatched bars represent elimination rates calculated from the model shown with the dashed lines (Fig. $3 E, F$; Eqs. $6-8)$. Error bars represent $95 \%$ confidence intervals.

mice was calculated to be $3.8 \%$, which is consistent with the measured rate of $4.1 \%$ (Fig. 6C). Similarly, for the $\mathrm{KO}$ mice, the predicted rate was $7.1 \%$, which is consistent with the measured rate of $8.5 \%$.

\section{Statistical distribution of spine volume}

Experimentally observed volume distributions in WT and $\mathrm{KO}$ mice were significantly different (KolmogorovSmirnov test, $p=0.003^{\mathrm{c}}$; Fig. $7 A-C$ ). Interestingly, small spines $\left(<0.06 \mu \mathrm{m}^{3}\right)$ in $\mathrm{KO}$ mice were more prevailing than those in WT mice, while the medium-sized spines were less abundant, and the very large spines (e.g., $>0.3 \mu \mathrm{m}^{3}$ ) tended to be more abundant in $\mathrm{KO}$ mice (Fig. 7C), although the difference was not statistically significant. Consequently, the average volume for the WT mice was $0.152 \pm 0.003 \mu \mathrm{m}^{3}$ (mean \pm SEM), and for the KO mice it was $0.144 \pm 0.003 \mu \mathrm{m}^{3}$, and these values were not significantly different ( $p=0.09$, Student's $t$ test).

We predicted the spine volume distribution from the volume dynamics of spines, as was done in a previous study (Yasumatsu et al., 2008). The values of $\sigma$ and $\mu$ were derived from the experimental data as the SD (Eq. 2) and the mean (Eq. 3) of the slow dynamics of the spine volume (Fig. $3 E, F$ ). The stationary distributions function
$f(V)$ of the spine volume could be calculated with Equation 11 (Risken, 1984), with a reflecting boundary condition at both the minimum $\left(V_{\min }=0.01 \mu \mathrm{m}^{3}\right.$; corresponding to the smallest spines observed) and maximum $\left(V_{\max }=1.0 \mu \mathrm{m}^{3}\right.$; only a few spines are larger than this) spine volumes. Figure $7, A(\mathrm{WT})$ and $B(\mathrm{KO})$, show that the calculated $f(V)$ (smooth lines) fits with the experimentally observed spine volume histograms (1368 spines for WT, 1913 spines for $\mathrm{KO}$ ). In particular, the model accounts for the abundance of small and large spines in $\mathrm{KO}$ mice (Fig. 7D), but not for the medium-sized spines, which is in line with the experimental observations. The model estimated the average spine volumes to be 0.134 and $0.137 \mu \mathrm{m}^{3}$ for WT and KO mice, respectively; these values were similar to the experimental observations.

\section{Discussion}

\section{Dynamics of spine volumes in the neocortex of WT and Fmr1 KO mice}

We performed in vivo imaging of dendritic spines in the adult mouse visual cortex using a resonant scan twophoton confocal microscope. The elimination rate of spines in the young hippocampus was $8 \% / d$ (Yasumatsu 
A
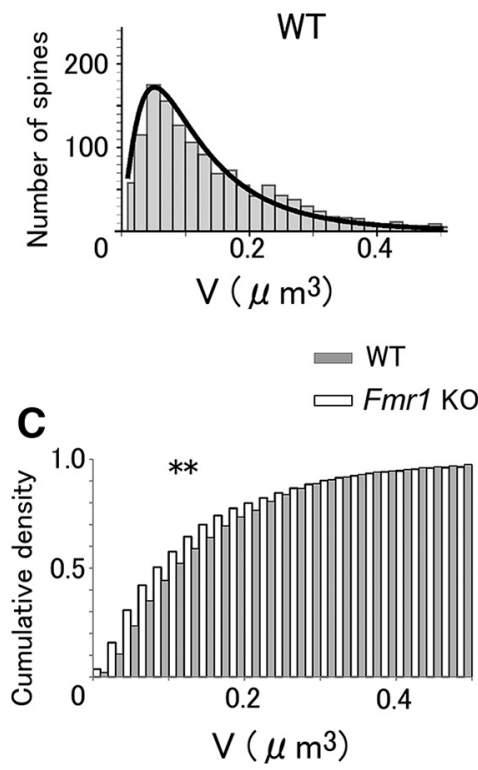

B

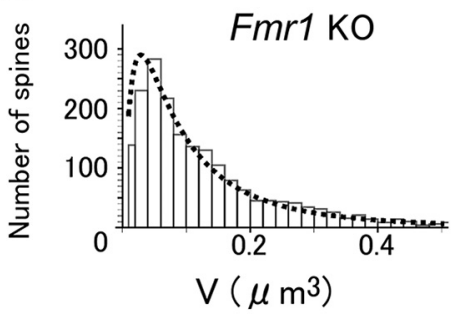

D

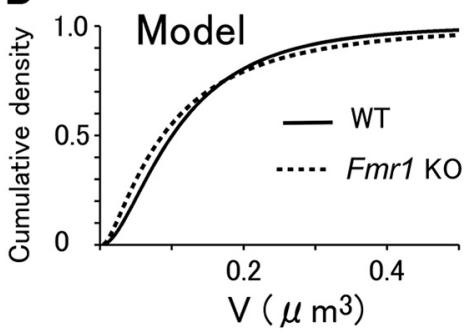

Figure 7. The volume distributions of spine-head volumes. $\boldsymbol{A}, \boldsymbol{B}$, The spine-head volume of WT $(\boldsymbol{A})$ and KO $(\boldsymbol{B})$ mice. The histogram represents data for 1368 spines $(\boldsymbol{A})$ and 1913 spines $(\boldsymbol{B})$. The lines represent a model fit by Equations 6,7 , and 11 based on dynamic data obtained (shown in Fig. 3E,F). C, D, Overlays of cumulative probability density distributions for WT and KO data (C) and models $(\boldsymbol{D})$. The volume distribution of KO mice data is significantly different from that of WT mice. $* * p=0.003^{\mathrm{C}}$ with the KolmogorovSmirnov test $\left(\chi^{2}=13.1, \mathrm{df}=2\right)$.

et al., 2008), but it was low in the neocortex (8\%/14 d; Zuo et al., 2005). Assuming the same linear model for $\sigma$, the fluctuations must be attenuated in the neocortex to 0.27 $(=1 / \sqrt{14})$ times the slope factor of that in young hippocampal preparations (Yasumatsu et al., 2008). We found, however, that when the values of $\sigma$ were fitted with linear functions of $V$, the slope factor was attenuated only to 0.75 times $[=0.21 / 0.28$, where 0.21 is the slope of the straight red line in Figure $3 A ; 0.28$ is the corresponding slope for the hippocampus from the previous report (Yasumatsu et al., 2008)]. This implies that the mathematical model developed for the hippocampus cannot be simply generalized to the neocortex. Instead, we found that the SD $\sigma$ of the spine volume dynamics was proportional to $V^{2 / 3}$, and not $V$, in the neocortex in vivo (Fig. 3E,F; Eqs. 6 and 7). Owing to the sublinearity, the actual values of $\sigma$ for small spines were smaller than those predicted from the linear fitting (Fig. $3 A, B$, red lines). Thus, the small spines in the neocortex are less dynamic than can be predicted from the linear (or multiplicative) dependence of the dynamics of spine volumes.

It is unclear why the values of $\sigma$ were proportional to the surface area $\left(V^{2 / 3}\right)$, while $\sigma$ depended linearly on $V$ in the young hippocampus. One reason for the variation in spine stability is that this factor may depend more on the extracellular matrix (ECM) in the adult mouse neocortex, and the surface structures of spines that contact the ECM play a more important role than do the cytosolic volume factors. Such stabilizing mechanisms are also active in Fmr1 $\mathrm{KO}$ mice, as the dynamics of their spines also fit our sublinear model. The slope factor of 0.28 in Fmr1 KO mice, compared with the slope factor of 0.20 in WT mice, could quantitatively account for the increases in the elim- ination rates of the spines. The unusually high expression of an ECM proteinase, matrix metalloproteinase-9, in Fmr1 KO mice may contribute to the greater slope factor observed in these mice (Sidhu et al., 2014; Reinhard et al., 2015; Nagaoka et al., 2016).

\section{Spine volume-related parameters in the neocortex of WT and Fmr1 KO mice}

Our in vivo study revealed that spine volumes showed apparently random fluctuations, akin to what would be observed if there were errors in the measurements, but the model developed using the volume dynamics well explained the observed values of volume distributions and spine turnovers in both WT and Fmr1 KO mice. This indicates that the observed dynamics of the spine volumes are the basis of the observed spine volume distributions. Interestingly, the spine volume distributions in Fmr1 KO and WT mice showed a complex dependence on the spine volume: small spines were more frequently observed in Fmr1 KO mice, but the medium-sized spines were less abundant. Moreover, large spines may be more abundant in Fmr1 KO mice than in their WT counterparts (Fig. 7C). This is the mathematical consequence of the larger slope factor in Fmr1 KO mice (Fig. 7D), which tends to sharpen the peak of the spine volume and then increase the tail of the distribution as a compensatory measure. Due to this secondary factor, the average spine volumes were similar (WT mice, $0.152 \mu \mathrm{m}^{3}$; KO mice, $0.144 \mu \mathrm{m}^{3}$ ). The subtle difference in the averaged volume could be one reason why spine volume changes are often overlooked (Nimchinsky et al., 2001; Wijetunge et al., 2014; but see Suresh and Dunaevsky, 2017). 
The complex nature of the effects of $F m r 1 \mathrm{KO}$ on spine volume distribution can be revealed only with a large sample size and adequate estimation of spine volumes using spine intensity in this and a previous study (Suresh and Dunaevsky, 2017), instead of spine width (Wijetunge et al., 2014). Despite the small difference in the average spine volume, the spine dynamics are severely impaired in Fmr1 KO mice even in adulthood, as is reflected in the increase in turnover rates (Pan et al., 2010; Padmashri et al., 2013; Nagaoka et al., 2016) and abnormal behaviors (Consortium-D-BFX, 1994; Padmashri et al., 2013).

In the present study, we did not distinguish between activity-dependent and activity-independent intrinsic dynamics (Yasumatsu et al., 2008; Minerbi et al., 2009; Kasai et al., 2010; Loewenstein et al., 2015; Nagaoka et al., 2016; Chambers and Rumpel, 2017; Mongillo et al., 2017; Okazaki et al., 2018; Ziv and Brenner, 2018). Since the spine elimination rates are not significantly affected by the blockage of NMDA receptors and voltage-dependent calcium channels under our conditions both in WT and Fmr1 KO mice (Nagaoka et al., 2016), it is possible that the slow dynamics measured here reflect activity-independent intrinsic dynamics to a large extent, if not completely. In the future, it will be interesting to test whether the increase in the slope factor is primarily due to the intrinsic dynamics, and to elucidate how the slope factor is determined.

\section{References}

Béïque JC, Lin DT, Kang MG, Aizawa H, Takamiya K, Huganir RL (2006) Synapse-specific regulation of AMPA receptor function by PSD-95. Proc Natl Acad Sci U S A 103:19535-19540. CrossRef Medline

Bhatt DH, Zhang S, Gan WB (2009) Dendritic spine dynamics. Annu Rev Physiol 71:261-282. CrossRef Medline

Chambers AR, Rumpel S (2017) A stable brain from unstable components: emerging concepts and implications for neural computation. Neuroscience 357:172-184. CrossRef Medline

Comery TA, Harris JB, Willems PJ, Oostra BA, Irwin SA, Weiler IJ, Greenough WT (1997) Abnormal dendritic spines in fragile $X$ knockout mice: maturation and pruning deficits. Proc Natl Acad Sci U S A 94:5401-5404. Medline

Consortium-D-BFX (1994) Fmr1 knockout mice: a model to study fragile $X$ mental retardation. The Dutch-Belgian Fragile $X$ Consortium. Cell 78:23-33. Medline

Cruz-Martín A, Crespo M, Portera-Cailliau C (2010) Delayed stabilization of dendritic spines in fragile $X$ mice. J Neurosci 30:77937803. CrossRef Medline

Fiala JC, Spacek J, Harris KM (2002) Dendritic spine pathology: cause or consequence of neurological disorders? Brain Res Rev 39:29-54. Medline

He CX, Portera-Cailliau C (2013) The trouble with spines in fragile $X$ syndrome: density, maturity and plasticity. Neuroscience 251: 120-128. CrossRef Medline

Herring BE, Nicoll RA (2016) Long-term potentiation: from CaMKII to AMPA receptor trafficking. Annu Rev Physiol 78:351-365. CrossRef Medline

Holtmaat A, Svoboda K (2009) Experience-dependent structural synaptic plasticity in the mammalian brain. Nat Rev Neurosci 10:647658. CrossRef Medline

Honkura N, Matsuzaki M, Noguchi J, Ellis-Davies GC, Kasai H (2008) The subspine organization of actin fibers regulates the structure and plasticity of dendritic spines. Neuron 57:719-729. CrossRef Medline
Kasai H, Fukuda M, Watanabe S, Hayashi-Takagi A, Noguchi J (2010) Structural dynamics of dendritic spines in memory and cognition. Trends Neurosci 33:121-129. CrossRef Medline

Lauterborn JC, Jafari M, Babayan AH, Gall CM (2015) Environmental enrichment reveals effects of genotype on hippocampal spine morphologies in the mouse model of fragile $X$ syndrome. Cereb Cortex 25:516-527. CrossRef Medline

Loewenstein Y, Kuras A, Rumpel S (2011) Multiplicative dynamics underlie the emergence of the log-normal distribution of spine sizes in the neocortex in vivo. J Neurosci 31:9481-9488. CrossRef Medline

Loewenstein Y, Yanover U, Rumpel S (2015) Predicting the dynamics of network connectivity in the neocortex. J Neurosci 35:1253512544. CrossRef Medline

Matsuzaki M, Ellis-Davies GC, Nemoto T, Miyashita Y, lino M, Kasai H (2001) Dendritic spine geometry is critical for AMPA receptor expression in hippocampal CA1 pyramidal neurons. Nat Neurosci 4:1086-1092. CrossRef Medline

Matsuzaki M, Honkura N, Ellis-Davies GC, Kasai H (2004) Structural basis of long-term potentiation in single dendritic spines. Nature 429:761-766. CrossRef Medline

Minerbi A, Kahana R, Goldfeld L, Kaufman M, Marom S, Ziv NE (2009) Long-term relationships between synaptic tenacity, synaptic remodeling, and network activity. PLoS Biol 7:e1000136. CrossRef Medline

Mongillo G, Rumpel S, Loewenstein Y (2017) Intrinsic volatility of synaptic connections-a challenge to the synaptic trace theory of memory. Curr Opin Neurobiol 46:7-13. CrossRef Medline

Moyer CE, Zuo Y (2018) Cortical dendritic spine development and plasticity: insights from in vivo imaging. Curr Opin Neurobiol 53: 76-82. CrossRef Medline

Mysore SP, Tai CY, Schuman EM (2007) Effects of N-cadherin disruption on spine morphological dynamics. Front Cell Neurosci 1:1. CrossRef Medline

Nagaoka A, Takehara H, Hayashi-Takagi A, Noguchi J, Ishii K, Shirai F, Yagishita S, Akagi T, Ichiki T, Kasai H (2016) Abnormal intrinsic dynamics of dendritic spines in a fragile $X$ syndrome mouse model in vivo. Sci Rep 6:26651. CrossRef Medline

Nimchinsky EA, Oberlander AM, Svoboda K (2001) Abnormal development of dendritic spines in FMR1 knock-out mice. J Neurosci 21:5139-5146. Medline

Noguchi J, Matsuzaki M, Ellis-Davies GC, Kasai H (2005) Spine-neck geometry determines NMDA receptor-dependent $\mathrm{Ca} 2+$ signaling in dendrites. Neuron 46:609-622. CrossRef Medline

Okazaki H, Hayashi-Takagi A, Nagaoka A, Negishi M, Ucar H, Yagishita S, Ishii K, Toyoizumi T, Fox K, Kasai H (2018) Calcineurin knockout mice show a selective loss of small spines. Neurosci Lett 671:99-102. CrossRef Medline

Padmashri R, Reiner BC, Suresh A, Spartz E, Dunaevsky A (2013) Altered structural and functional synaptic plasticity with motor skill learning in a mouse model of fragile $X$ syndrome. $J$ Neurosci 33:19715-19723. CrossRef

Pan F, Aldridge GM, Greenough WT, Gan WB (2010) Dendritic spine instability and insensitivity to modulation by sensory experience in a mouse model of fragile $X$ syndrome. Proc Natl Acad Sci U S A 107:17768-17773. CrossRef Medline

Paxinos GF (2001) The mouse brain in stereotaxic coordinates, Ed 2. San Diego: Academic.

Penzes P, Cahill ME, Jones KA, VanLeeuwen JE, Woolfrey KM (2011) Dendritic spine pathology in neuropsychiatric disorders. Nat Neurosci 14:285-293. CrossRef Medline

Purpura DP (1974) Dendritic spine "dysgenesis" and mental retardation. Science 186:1126-1128. Medline

Reinhard SM, Razak K, Ethell IM (2015) A delicate balance: role of MMP-9 in brain development and pathophysiology of neurodevelopmental disorders. Front Cell Neurosci 9:280. CrossRef Medline

Risken H (1984) The Fokker-Planck equation: methods of solution and applications. Berlin; New York: Springer. 
Rudelli RD, Brown WT, Wisniewski K, Jenkins EC, LaureKamionowska M, Connell F, Wisniewski HM (1985) Adult fragile X syndrome. Clinico-neuropathologic findings. Acta Neuropathol 67: 289-295. Medline

Sidhu H, Dansie LE, Hickmott PW, Ethell DW, Ethell IM (2014) Genetic removal of matrix metalloproteinase 9 rescues the symptoms of fragile X syndrome in a mouse model. J Neurosci 34:98679879. CrossRef

Sidorov MS, Auerbach BD, Bear MF (2013) Fragile X mental retardation protein and synaptic plasticity. Mol Brain 6:15. CrossRef Medline

Statman A, Kaufman M, Minerbi A, Ziv NE, Brenner N (2014) Synaptic size dynamics as an effectively stochastic process. PLoS Comput Biol 10:e1003846. CrossRef Medline

Suresh A, Dunaevsky A (2017) Relationship between synaptic AMPAR and spine dynamics: impairments in the FXS mouse. Cereb Cortex 27:4244-4256. CrossRef Medline

Swanger SA, Yao X, Gross C, Bassell GJ (2011) Automated 4D analysis of dendritic spine morphology: applications to stimulus- induced spine remodeling and pharmacological rescue in a disease model. Mol Brain 4:38. CrossRef

Wijetunge LS, Angibaud J, Frick A, Kind PC, Nagerl UV (2014) Stimulated emission depletion (STED) microscopy reveals nanoscale defects in the developmental trajectory of dendritic spine morphogenesis in a mouse model of fragile $X$ syndrome. $J$ Neurosci 34:6405-6412. CrossRef

Yasuda R (2017) Biophysics of biochemical signaling in dendritic spines: implications in synaptic plasticity. Biophys J 113:21522159. CrossRef Medline

Yasumatsu N, Matsuzaki M, Miyazaki T, Noguchi J, Kasai H (2008) Principles of long-term dynamics of dendritic spines. J Neurosci 28:13592-13608. CrossRef Medline

Ziv NE, Brenner N (2018) Synaptic tenacity or lack thereof: spontaneous remodeling of synapses. Trends Neurosci 41:89-99. CrossRef Medline

Zuo Y, Yang G, Kwon E, Gan WB (2005) Long-term sensory deprivation prevents dendritic spine loss in primary somatosensory cortex. Nature 436:261-265. CrossRef Medline 